\title{
Preparation of nanocellulose from jute fiber waste
}

\begin{abstract}
A significant amount of waste fibers is produced in the form of short length from textile spinning industry during several mechanical processes. The objective of this study was to utilize the waste from spinning of fibers in the form of short fibers for the production of nano scale fillers in the applications of nanocomposite. This work concerns with fibers of jute as the raw material to produce nano cellulose with the help of high energy ball milling technique and its potential applications as fillers in biodegradable nanocomposite plastics used in automotives, packing and agriculture applications. Influence of various milling conditions like nature of milling that is dry or wet, milling time and ball size are studied on the particle size distribution and morphology of jute nanoparticles obtained. Wet milling in the deionized water resulted into particle size refinement below $500 \mathrm{~nm}$ with comparatively thin size distribution after $180 \mathrm{~min}$ of ball milling at the cost of small amount of contaminations introduced from milling media.
\end{abstract}

Volume 4 Issue I - 2018

Rehan Abbasi, ' Vijay Baheti²

'Department of Textile Engineering, BUITEMS, University in

Quetta, Pakistan

${ }^{2}$ Technical University of Liberec, Czech Republic

Correspondence: Rehan Abbasi, Associate Professor, Department of Textile Engineering, BUITEMS, University in Quetta, Quetta, Pakistan, Tel 0333-7834616, Email rehan_abbaci@hotmail.com

Received: January 22, 2018 | Published: February 22, 2018

Keywords: high energy ball milling, particle size, contamination

\section{Introduction}

The natural fibers are cheap and biodegradable materials. They have relatively high aspect ratio, high strength to weight ratio, reasonable low density and good to moderate tensile strength and initial modulus. These characteristics make these fibers an attractive class of textile materials which traditionally used for production of yarn by spinning. However, due to particular limitations of the spinning process, the shorter fibers are not suitable for efficient production and therefore result into the waste. Therefore, numerous methods are being discovered to use short waste fibers as they hold some useful inherent properties. ${ }^{1-3}$ One such fascinating approach is to isolate the nano fibrils or nano crystals from the short fibers and use it as the fillers in preparation of nanocomposites. The examples of such techniques to separate the fibrils or crystals from the waste fibers are steam explosion, cryo-crushing, high pressure turbulence and shear grinding, etc. ${ }^{4-6}$

This study emphasises upon utilization of jute fiber wastes to produce nanocellulose by combined action of chemical treatment and high energy planetary ball milling process. The compositions of jute fibers include mainly cellulose, hemicellulose and lignin. The chemical treatment removes lignin and hemicellulose from jute fibers and defibrillates the fiber structure. The ball milling process further grinds the material into powder form to separate nanowhiskers of jute which is nothing but nanocellulose. The obtained nanocellulosic structure from jute fibers is expected to show high stiffness and thus the reinforcement of nanocellulosic powder into polymer matrix provides the value added materials with superior performance. The potential applications of these nanocomposites are in biodegradable plastics used in automotive, packaging and agriculture. ${ }^{7,8}$

The planetary ball mill is the most commonly used technique for breaking down the solid materials mechanically. The system is predominantly suitable for research purposes since it utilizes very small amount of material. The planetary ball mill technique comprises of one turn disc and 2 or 4 containers. This disc revolves in one direction while the containers revolve in the opposite direction. The centrifugal force, produced by the rotation of the container around its own axis combined with the rotation of the turn disc, are realized to the milled substrate and milling balls in the container.

The movement of the milling balls and the milled substrate is illustrated in Figure 1. Since the container and turn disc have opposite direction of rotation, the centrifugal forces are consecutively harmonised.

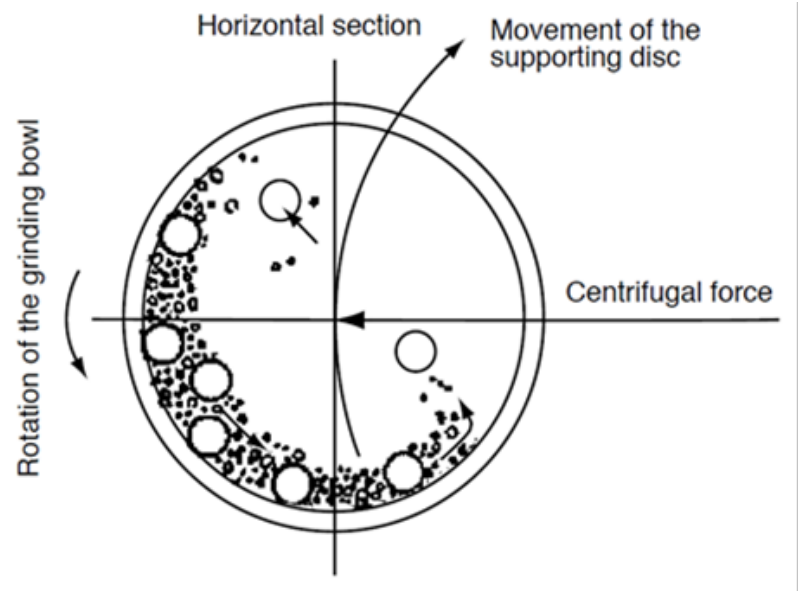

Figure I Working principle of planetary ball milling.

Consequently rubbing resulted from the tough milling balls and the substrate being milled interchangeably rolling on the inner wall of the container and striking the opposite wall. Therefore, the planetary ball mill is used for high speed milling.

\section{Materials and methods}

Initially the fibers were cut in $30 \mathrm{~mm}$ length to prepare small segments. Chemical treatment of jute fibers was then carried out to 
eliminate the hemicellulose and lignin. Jute fibers were successively undergone for mercerization by $18 \%$ Sodium Hydroxide at room temperature for $120 \mathrm{~min}$, then $1 \mathrm{M}$ Sulfuric acid treatment at $80^{\circ} \mathrm{C}$ for $60 \mathrm{~min}$ and subsequently with $4 \%$ Sodium Hydroxide treatment at $80^{\circ} \mathrm{C}$ for further $60 \mathrm{~min}$ as indicated in Figure 2.,

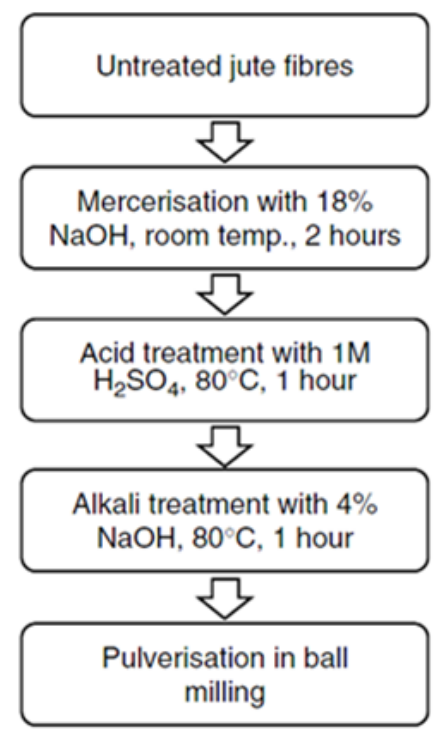

Figure 2 Chemical treatment of jute fibers.

After chemical treatment, grinding of jute fibers was carried out by a planetary ball milling machine Pulverisette 7 manufactured by Fritsch ${ }^{\circledR} \mathrm{GmbH}$ in a sintered corundum bowl by using zirconia balls having diameter of $10 \mathrm{~mm}$ initially for $10 \mathrm{~min}$ for dry milling and
$3 \mathrm{~mm}$ diameter for further $180 \mathrm{~min}$ of milling in deionized water. The container was loaded with fibers keeping ball to material ratio (BMR) of 10:1 and the speed of rotation of the container was set at $850 \mathrm{rpm}$.

The particle size measurement of the milled material was carried out on Zetasizer nano series by Malvern ${ }^{\circledR}$ after each 60 min of ball milling. The dispersion medium was deionised water. The dispersion was ultrasonicated for $5 \mathrm{~min}$ before characterization whereas the refractive index was set at 1.52 to analyse the particle size.

Single fiber strength of untreated jute fiber and chemically treated jute fiber was measured on vibroskop/vibrodyn by Lenzing technik ${ }^{\circledR}$ with jaw speed of $10 \mathrm{~mm} / \mathrm{min}$, gauge length of $10 \mathrm{~mm}$ and tension weight of $500 \mathrm{mg}$. Hence, in total 50 observations were recorded and mean value was calculated.

Morphological study of the jute fibers after each phase of chemical treatment and ball milling was witnessed on scanning electron microscope (SEM) by TESCAN VEGA ${ }^{\circledR}$ at $30 \mathrm{KV}$.

Fourier Transform Infra Red (FTIR) analysis was carried out to confirm the removal of lignin after chemical treatment and introduction of contamination in the jute powder due to milling process.

\section{Results and discussion}

\section{Influence of treatment of chemicals on single fiber strength}

Mechanical properties of untreated jute fibers and after treatment with chemicals are shown in Figure 3 as well as in Table 1. Significant loss on modulus and tenacity was observed after chemical treatment. The reason was acid hydrolysis which was responsible for rupture of the bonds leading to deterioration in tensile strength considered to be useful in easier milling of the fibers.

Table 1 Mechanical properties of jute fibres

\begin{tabular}{lllll}
\hline Fibre Type & Linear Density (dtex) & Young's Modulus (g/den) & Tenacity (CN/tex) & Elongation \% \\
\hline Untreated Jute & 14.99 & 227.2 & 56.32 & 2 \\
Treated Jute & 14.69 & 30.16 & 28.39 & 6.98 \\
\hline
\end{tabular}

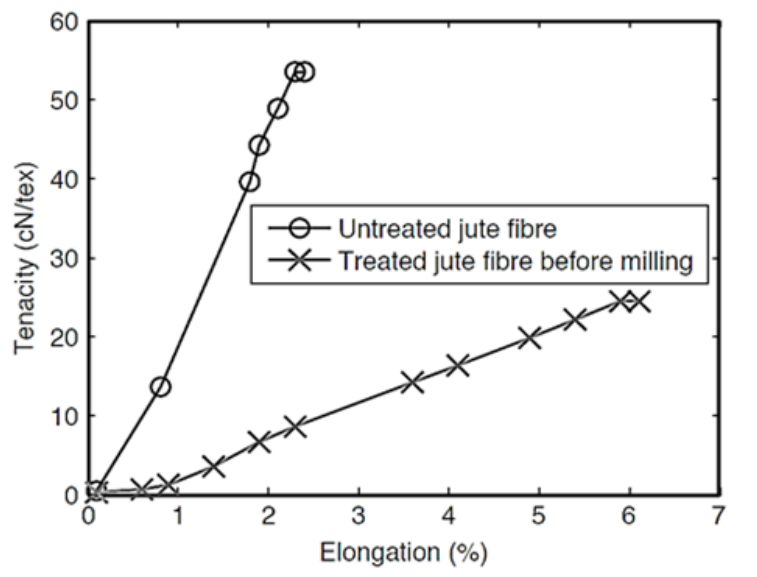

Figure 3 Single fiber strength test.

\section{Influence of treatment of chemicals on morphology}

The morphological derivations in jute fibers after each chemical treatment are presented in Figure 4. The jute fibers before treatment were bundles of individual strands held together by lignin (Figure 4A) but after mercerization and acid treatment, lignin was cleaned off partly and defibrillated the structure (Figures 4B) (Figure 4C). After treatment with alkaline solution at $80^{\circ} \mathrm{C}$, a number of micro fibrils came out due to axial splitting of the fiber structure (Figure 4D).

\section{Influence of chemical treatment on structure of the fiber}

The FTIR spectrums of untreated and chemically treated jute fibers are presented in Figure 5. The typical peaks of lignin and cellulose lie at about $1000 \mathrm{~cm}^{-1}$ (due to C-O stretching vibration of the cellulose molecule) and $1500-1600 \mathrm{~cm}^{-1}$ (due to aromatic skeleton vibration of the lignin) respectively. It was observed that the characteristic peaks were situated in the same position after each treatment of chemical. However, the relative intensities of the peaks varied significantly. The 
ratio of $1500-1600 \mathrm{~cm}^{-1}$ to $1000 \mathrm{~cm}^{-1}$ increased, which confirmed that the content of cellulose increased and the content of lignin decreased.

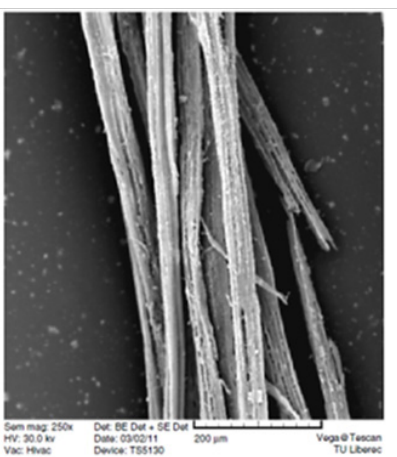

(a) Untreated

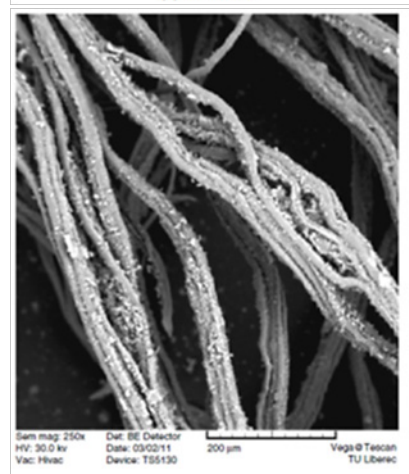

(c) Acid treatment

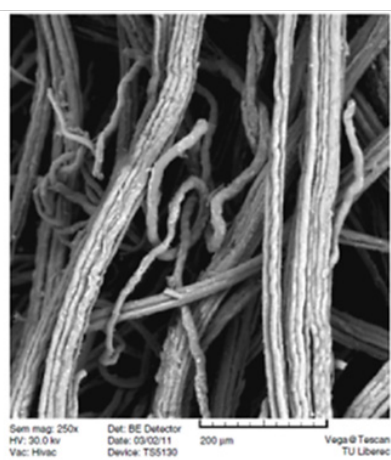

(b) Mercerisation

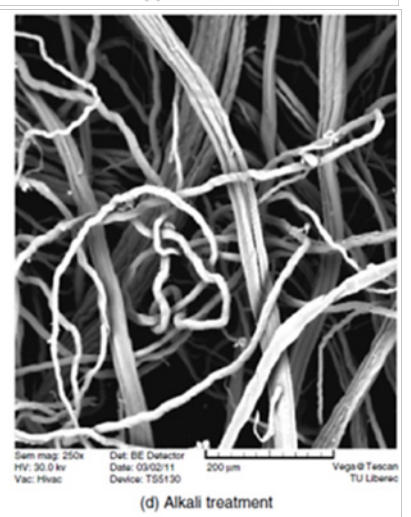

(d) Alkali treatmen
Figure 4 Morphology of jute fibers.

\section{Influence of milling condition on particle size}

The results of particle size distribution and the morphology of jute cellulose nanowhiskers after 60 min milling in dry and wet conditions are shown in Figures 6,7 respectively. Very wide particle size distributions were observed in the case of dry milling. The reason was sticking of material to the surface of container after certain duration of milling below which further refinement was slow.

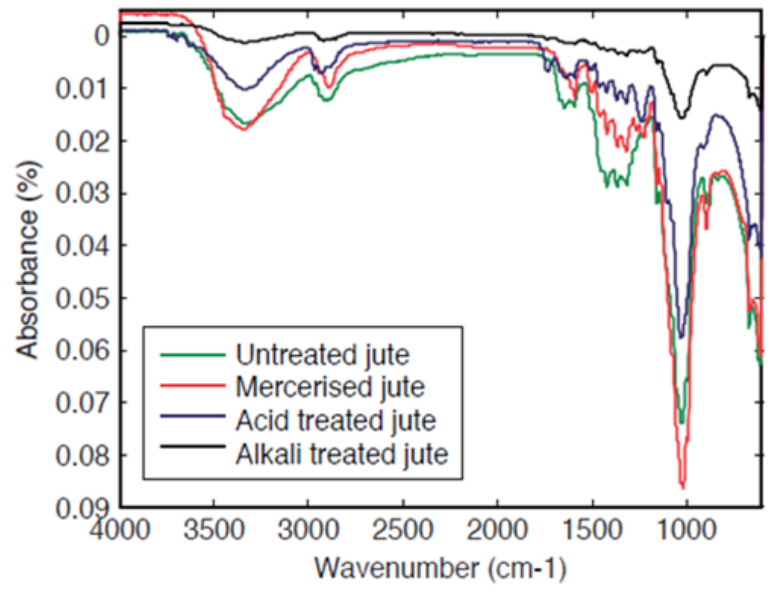

Figure 5 FTIR analysis of jute fibers after each chemical treatment.
This happened due to dominant role played by surface forces in dry state leading to the deposition of material on the surface of milling media and aggregation. While this phenomenon was not observed during wet milling conditions resulted in narrow size distribution. The general practice of addition of process active agents in milling is to suppress the role of surface forces and thus to prevent the agglomeration tendency to get narrow particle size distribution. However our study was aimed without the usage of any such agent. Therefore, we chose the wet milling conditions for further refinement of jute fibres under extended duration.

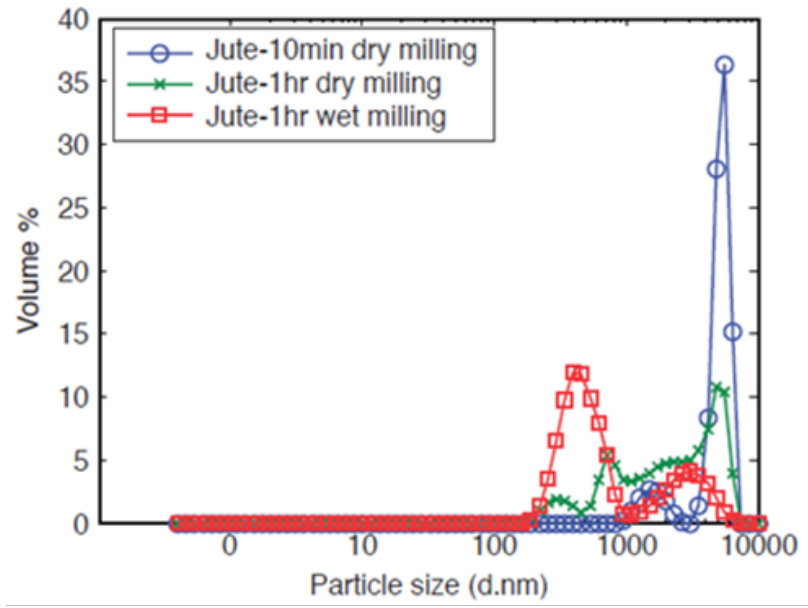

Figure 6 Influence of milling condition on particle size reduction.

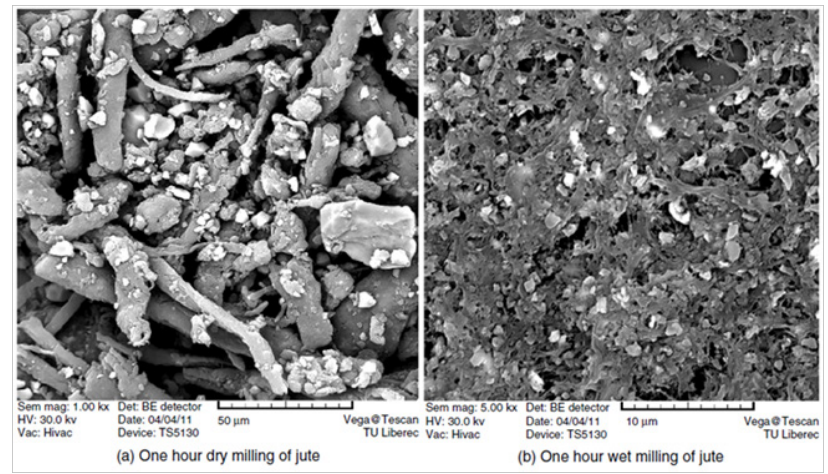

Figure 7 SEM micrographs of milled powder.

Influence of milling time (in wet condition) on particle size

As wet milling did not encounter the problem of sticking to the container, so it was carried out further for $180 \mathrm{~min}$. Figure 8 shows particle size distribution results for every $60 \mathrm{~min}$ of wet milling. It was observed that as wet milling time increased, the bimodality in distribution reduced giving narrower size distribution results. Though the results show that particle size is around $500 \mathrm{~nm}$ and not below $100 \mathrm{~nm}$, still we consider it satisfactory. The reason is that it may possible to achieve the particle size below $100 \mathrm{~nm}$ with longer wet milling time, higher ball to material ratio, addition of process active agents, higher milling speed etc. However there is always possibility of finding contaminations coming from milling media into the material under longer milling time. Hence balance must be done 
between particle size distributions and level of contaminations during ball milling.

\section{Influence of milling process on contamination of milling powder dispersion}

FTIR analysis from Figure 9 confirmed that the chemical composition of jute powder dispersion obtained after $180 \mathrm{~min}$ of wet milling was the same as untreated and chemically treated jute fiber Thus the dispersion was of nanocellulose with small percentage of inorganic contaminations introduced from milling media responsible for different spectra below $1000 \mathrm{~cm}^{-1}$ wave number.

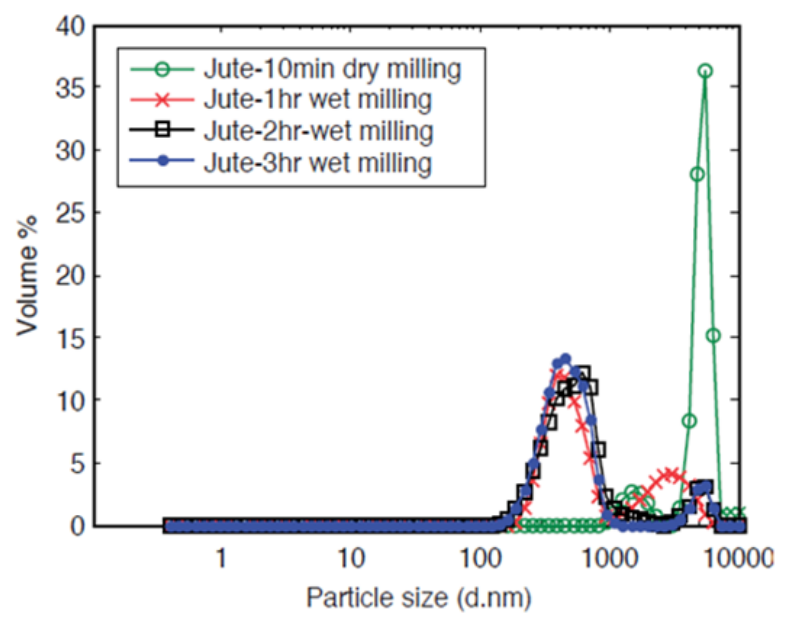

Figure $\mathbf{8}$ Influence of wet milling time on particle size distribution.

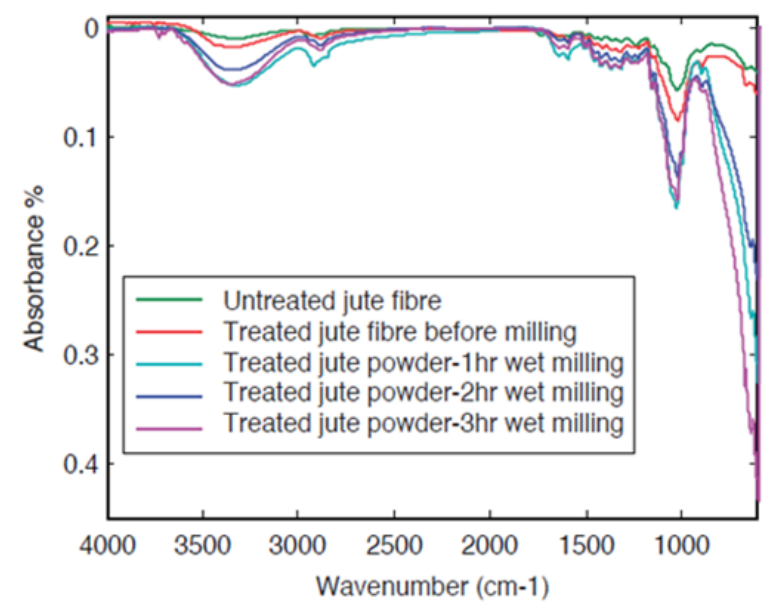

Figure 9 FTIR analysis of jute powder dispersion after wet milling.

\section{Conclusion}

Milling is the unique technique to produce nanoparticles of any kind of material like waste of textile fibers. It is simple in operation and universal in nature. This technique has the plenty of scope to become industrially applicable as it can produce nanoparticles in large quantity.

Jute fibers were successfully pulverized into powder dispersion form below $500 \mathrm{~nm}$ with relatively thin size distribution after 180 min of milling in wet condition with the help of deionized water. Dry milling was difficult due to the sticking of material to milling media. FTIR results confirmed small percentage of inorganic contaminations introduced from milling media in the dispersion.

Further modifications in wet milling process like addition of process active agent, increased ball to material ratio, smaller size of balls, increased milling time, etc need to be studied to effectively reduce the particle size below $100 \mathrm{~nm}$.

\section{Acknowledgement}

There is no acknowledgement.

\section{Conflict of Interest}

There is no conflict of interest in publishing the article.

\section{References}

1. Yuen C, Cheng Y, Li Y, et al. Preparation and characterisation of nanoscale cotton powder. J Textile Institute. 2009;100(2):165-172.

2. Prasad BM, Sain MM, Roy DN. Properties of ball milled thermally treated hemp fibers in an inert atmosphere for potential composite reinforcement. J Material Science. 2005;40(16):4271-4278.

3. Chang F, Lee SH, Toba K, et al. Bamboo nanofiber preparation by $\mathrm{HCW}$ and grinding treatment and its application for nanocomposite. Wool Sci Technol. 2011;46(1-3):1-11.

4. Jahan MS, Abrar S, Zhibin H, et al. Jute as raw material for the preparation of microcrystalline cellulose. Cellulose. 2010;18(2):451459.

5. Moran JI, Alvarez VA, Cyras VP, et al. Extraction of cellulose and preparation of nanocellulose from sisal fibers. Cellulose. 2008;15(1):149-159.

6. Siro I, Plackett D. Microfibrillated cellulose and new nanocomposite materials: a review. Cellulose. 2010:17(3):459-494.

7. Aulin C, Gallstedt M, Lindstrom T. Oxygen and oil barrier properties of microfibrillated cellulose films and coatings. Cellulose. 2010;17(3):559 574

8. Garcia M, Lagaron JM. On the use of plant cellulose nanowhiskers to enhance the barrier properties of polylactic acid. Cellulose. 2010;17(5):987-1004.

9. Wang H, Huang L, Yafei L. Preparation and characterisation of micro and nano fibrils from jute. Fibers and Polymers. 2009;10(4):442-445. 